\title{
Comparison of the diagnostic and predictive potential of three anti-citrullinated protein antibodies as adjuncts to rheumatoid factor and CCP in a cohort of South African rheumatoid arthritis patients
}

Pieter W.A. Meyer ${ }^{1}$, Mahmood T.M. Ally ${ }^{2}$, Bridget Hodkinson ${ }^{3}$, Ronald Anderson ${ }^{4}$ and Mohammed Tikly ${ }^{3}$.

\footnotetext{
${ }^{1}$ Department of Immunology, Faculty of Health Sciences, University of Pretoria, South Africa, and Tshwane Academic Division of the National Health Laboratory Service, Pretoria, South Africa.

${ }^{2}$ Department Internal Medicine, Faculty of Health Sciences, University of Pretoria, Pretoria, South Africa, and Steve Biko Academic Hospital, Pretoria, South Africa.

${ }^{3}$ Division of Rheumatology, Department of Internal Medicine, Faculty of Health Sciences, University of the Witwatersrand, Johannesburg, and Chris Hani-Baragwanath Academic Hospital, Soweto, South Africa.

${ }^{4}$ Department of Immunology, Faculty of Health Sciences, University of Pretoria, Pretoria, South Africa.
}

\section{Corresponding Author:}

Dr Pieter WA Meyer

Address: Department Immunology, Pathology Building, 5 Bophelo Rd, University of Pretoria, Prinshof Campus, Pretoria, South

Africa.

E mail: $\quad$ pieter.meyer@up.ac.za

ORCID: 0000-0002-7318-1055

Telephone: + +27 123192977

Fax: $\quad$ +27 $\quad+23230732$

\begin{tabular}{|l|l|l|}
\hline Co-author & Emails: & $\underline{\text { ORCIDs }}$ \\
\hline Mahmood T.M. Ally & $\underline{\text { mahmoodally@gmail.com }}$ & None \\
\hline Bridget Hodkinson & $\underline{\text { drbridget@gmail.com }}$ & None \\
\hline Ronald Anderson & $\underline{\text { ronald.anderson@up.ac.za }}$ & $0000-0002-5925-6452$ \\
\hline Mohammed Tikly & $\underline{\text { tiklymohammed01@gmail.com }}$ & None \\
\hline
\end{tabular}

\section{Compliance with Ethical Standards:}

Funding: This study was funded by departmental funds of the Rheumatology and Immunology Departments involved, no grant funds were used.

\section{Conflict of interest:}

None of the authors have any conflict of interest to disclose.

\section{Ethical Approval:}

All procedures performed in this study involving human participants were in accordance with ethical standard of the Universities of Witwatersrand (WITS) and Pretoria's (UP) ethical research committees and the Helsinki declaration of 1964 and its later amendments. Ethics certificate reference numbers: 09/2017 (UP, issued 30/01/2017) and M150513 (WITS, issued 29/09/2015). 


\title{
Informed Consent:
}

Informed, and signed consent was obtained from each individual participant included in this study as per the institutions Ethics Boards requirement.

\begin{abstract}
:
Purpose:

A retrospective comparison of the prevalence and diagnostic value of anti-Sa, anti-CEP-1, and anti-MCV autoantibodies relative to those of the established autoantibodies, composite RF and anti-CCP-IgG used routinely for RA diagnosis as a component of the ACR 2010 criteria, in a cohort of disease-modifying antirheumatic drug naïve African RA patients $(n=75)$.
\end{abstract}

Methods:

Serum concentrations of anti-Sa, anti-CEP-1 and anti-MCV autoantibodies were measured using ELISA procedures, while anti-CCP-IgG antibodies were determined by fluorescence enzyme immunoassay, and composite RF by latex-enhanced laser nephelometry.

Results:

The seropositivity frequencies of anti-Sa, anti-CEP-1 and anti-MCV antibodies for the RA patients were $82 \%, 72 \%, 85 \%$ respectively, while that of anti-CCP-IgG and RF was $87 \%$ for both. Overall, anti-MCV demonstrated the best specificity, positive predictive value (PPV), odds ratio and positive likelihood ratio of all the types of autoantibody tested.

\section{Conclusion:}

These observations in this unique cohort of RA patients indicated novel associations of all 3 autoantibodies in regard to HLA-SE risk alleles, disease severity and tobacco use that was not reported before. Elevated anti-Sa titers designated a propensity of higher disease and high-risk alleles in our cohort. Anti-CEP-1 association with HL|A-SE homozygosity and high risk alleles is also novel in this group. Of note, measurement of antiMCV antibodies on presentation, either as an adjunctive or even as a stand-alone test, surpassed all other biomarkers investigated here and therefore may add value to clinical management.

\section{Keywords:}

Biomarkers; anti-citrullinated peptide antibodies; Anti-CCP; Anti-Sa; Anti-CEP-1; anti-MCV 


\section{Introduction}

Rheumatoid arthritis (RA) is a chronic inflammatory disease affecting the joints, leading to cartilage destruction and debilitating disease. Worldwide, RA affects 1-3\% of the general population with females in the majority. Composite (IgA, IgG, IgM) rheumatoid factor (cRF) and anti-citrullinated peptide (anti-CCPIgG) antibodies are components of the 2010 ACR/EULAR RA classification criteria, underscoring the serodiagnostic utility of these autoantibodies in RA [1]. The efficacy of this strategy in detecting early disease, thereby improving prognosis, is well-established worldwide [2-5], and includes black South African RA patients [5-9]. However, less is known about the diagnostic and prognostic potential of measurement of the more recently described autoantibodies, anti-Sa, anti-CEP-1and anti-MCV in African patients with RA, as well as their associations with prominent disease risk factors, specifically tobacco usage and positivity for the human leucocyte antigen - shared epitope (HLA-SE).

The clinical utility of measurement of a group of ACPAs namely, anti-Sa, anti-CEP-1 and anti-MCV autoantibodies was evaluated in the current study. Serum levels and prevalence rates were compared with those of traditional anti-CCP-IgG and cRF autoantibodies in a cohort of disease-modifying, anti-rheumatic drug (DMARD)-naïve, black, predominantly female South African patients with RA. Furthermore, the sensitivities, specificities and normal threshold values of these ACPAs were also determined by inclusion of measurement in serum from healthy individuals and patients with systemic lupus erythematosus (SLE) or scleroderma.

\section{Patients \& Methods}

The study, which conformed to good clinical and laboratory practice and to the Helsinki Declaration, was approved by the Research Ethics Committees of the Faculties of Health Sciences of the Universities of the Witwatersrand and Pretoria.

The cohort consisted of a group ( $n=75)$ of black African RA patients collected from 2010 that formed part of a previously described cohort, all diagnosed according to the 1987 ACR RA criteria and who were DMARDnaïve at baseline. All patients were started on methotrexate regimen with some on additional corticosteroids. [6] Methods for serological measurement of cRF and anti-CCP-IgG have been described previously for this cohort of RA patients [6-8]. Sample aliquots was stored at $-80^{\circ} \mathrm{C}$ to prevent deterioration of antibodies.

Controls ( $\mathrm{n}=75$ ) consisted of 20 SLE patients, 26 scleroderma patients and 29 healthy individuals. Due to limited access to patients with other autoimmune diseases as well as budget consideration the control composition was composed as above to at least match the patient group. The fact that other autoimmune diseases known to react to these autoantibodies is not represented in the control group is a limitation and should be taken into consideration. We do however consider that the most common of autoimmune disorders in SLE and scleroderma is an advantage instead of normal healthy controls. 
The standard classification for SDAI (Simplified Disease Activity Index)) was used [remission $=\leq 3$, low disease activity $($ LDA $)=>3-\leq 11$, mild disease activity $(\mathrm{MDA})=>11-\leq 26$, and high disease activity (HDA) >26].[9] DAS (Disease Activity Score)28-3 scores were classified according to Fransen et al. [10] Disease duration since onset of symptoms is expressed in months and was categorised as: i) $<6$ months ( $n=24)$, ii) between 6 and 12 months $(\mathrm{n}=27)$ and, iii $)>12$ months $(\mathrm{n}=24)$.

ELISA procedures were used to measure serum concentrations of anti-Sa (IU/ml) and anti-CEP-1(U/ml) (EUROIMMUN AG, Luebeck, Germany), anti-MCV (U/ml) (Orgentec Diagnostika GmbH, Mainz, Germany), and cotinine (ng/ml) as a marker of tobacco usage (Calbiotech Inc., Spring Valley, CA, USA). Composite RF (IU/ml) and high sensitivity (hs)-CRP (mg/dl) were determined using latex-enhanced lasernephelometry (Siemens South Africa, Midrand, South Africa), while anti-CCP-IgG (EliA U/ml) was determined by fluorescence enzyme immunoassay (FEIA) using the automated Phadia 250 platform (Phadia AB, Uppsala, Sweden).

Both the molecular procedure used for HLA-DRB1 typing and categorisation of HLA-SE genotype have been described previously [7].The HLA-SE classification was validated according to the RAA amino acid sequence at positions 72-74 of the third hypervariable region of the HLA-DRB1 gene and assigned SS or SX if both HLA-DRB1 alleles or only 1 allele expressed the RAA motif respectively. Modulation of risk according to the types of amino acids at positions 70 and 71 of the third hypervariable region of the HLA-DRB1 gene enabled further subdivision into HLA-SE low-risk (LR) and high-risk (HR) alleles.[11, 12]

\section{Statistical analysis}

Descriptive and inferential statistics techniques were used in the analyses. Tests for association of contingency tables were performed using two-tailed $\mathrm{Chi}^{2}$ or Fisher's exact tests. One-way ANOVA and both paired and unpaired t-tests were performed after skewed data was log transformed. Correlation coefficients were derived from correlation matrices using Bonferroni's method $p$ value correction for multiple testing. The empirical estimation of cut-off points for diagnostic tests was performed using Phil Clayton's (ANZDATA Registry, Australia) STATA program 'cutpt' which is based on ROC (receiver operator curve) analysis. We used 'cutpt' with the Liu method which maximises the product of the sensitivity and specificity. The estimated cut-off point returned was bootstrapped to estimate confidence intervals. Statistical significance was determined at a p-value $<0.05$ and confidence intervals of $95 \%$. The analyses were performed using STATA 13, Statacorp, Texas, USA. 


\section{Results}

\section{Demographics}

The demographics and HLA-SE related data of the group of RA patients are summarised in Table 1. The median group age was 48 years, with a median SDAI score of 40, DAS28-3 of 5.5 and disease duration of 9 months. Eighty-one percent were female, and 25 percent used tobacco products. Of the control groups, the SLE and Scleroderma groups were age and gender compatible with median ages recorded of 43 and 45 years respectively, and predominantly female. The healthy group was age match (median age 46) but with a gender ratio of 2:1 (19 female: 11 males). Seropositivity rates of the different test autoantibodies for patients and control groups as shown in Table 2. Anti-MCV had the lowest positivity rate in the control group, whilst anti-CEP-1 was the highest number of false positives in the control groups.

Table 1. Demographic, clinical and biomarker profiles (medians and iqr values) of the cohort of RA patients

\begin{tabular}{|ccc|}
\hline \hline & Median (iqr) & $n(\%)$ \\
& i.e. above cut-off or elevated \\
\hline Age (years) & Demographics & \\
Baseline SDAI score & $48(19)$ & \\
Baseline DAS28-3 & $40(25)$ & $61(81)$ \\
Duration (months) & $5.5(1.8)$ & $34(45)$ \\
Females & $9(12)$ & $58(77)$ \\
Tobacco use & & $41(55)$ \\
hs-CRP ( $\mu$ g/ml) & $13(22)$ & $30(40)$ \\
& & $4(5)$ \\
Homozygous (SS) & & $50(70)$ \\
Heterozygous (SX) & & $21(30)$ \\
None (XX) & & \\
High-risk-associated alleles (HR) & & \\
Low-risk-associated alleles (LR) & & \\
\hline
\end{tabular}

SDAI Simplified Disease Activity Index, DAS-28-3 Disease Activity Score, CRP C-reactive protein

Table 2. Medians, interquartile ranges (iqr) and positivity rates of the test autoantibodies in patients with RA, SLE, or scleroderma, as well as healthy control subjects

\begin{tabular}{|c|c|c|c|c|c|c|c|c|}
\hline \multirow[b]{2}{*}{ Autoantibodies } & \multicolumn{2}{|c|}{ RA $(n=75)$} & \multicolumn{2}{|c|}{ SLE $(n=20)$} & \multicolumn{2}{|c|}{ Scleroderma $(n=26)$} & \multicolumn{2}{|c|}{ Healthy $(n=29)$} \\
\hline & $\begin{array}{c}\text { p50 } \\
\text { (iqr) }\end{array}$ & $n(\%)^{\mathrm{a}}$ & p50 (iqr) & $n(\%)^{\mathrm{a}}$ & p50 (iqr) & $n(\%)^{\mathrm{a}}$ & p50 (iqr) & $n(\%)^{\mathrm{a}}$ \\
\hline cRF (IU/ml) & $\begin{array}{c}161 \\
(403)\end{array}$ & $65(87)$ & $10(0)$ & $3(11)$ & $10(0)$ & $3(12)$ & $10(0)$ & $1(3)$ \\
\hline Anti-CCP (EliA U/ml) & $\begin{array}{c}494 \\
(839)\end{array}$ & $65(87)$ & $6(7)$ & $6(29)$ & 2(2) & $2(7)$ & $6(7)$ & $1(3)$ \\
\hline Anti-Sa (IU/ml) & $\begin{array}{c}119 \\
(203)\end{array}$ & $61(81)$ & $0(2)$ & $3(14)$ & $0(1)$ & $2(7)$ & $0(3)$ & $3(13)$ \\
\hline Anti-CEP-1 (U/ml) & 75 (169) & $54(72)$ & $45(44)$ & $11(57)$ & $25(26)$ & $5(19)$ & $20(12)$ & $1(3)$ \\
\hline Anti-MCV (U/ml) & $\begin{array}{c}352 \\
(809)\end{array}$ & $64(85)$ & $39(43)$ & 1(3) & $39(26)$ & $1(4)$ & $27(16)$ & $0(0)$ \\
\hline
\end{tabular}

p50-median

$c R F$ composite rheumatoid factor, $C C P$ cyclic citrullinated peptide (IgG), $C E P-1$ citrullinated $\alpha$-enolase peptide-1, $M C V$ mutated cyclic vimentin, $R A$ rheumatoid arthritis, SLE systemic lupus erythematosus 


\section{Validation of cut-off values}

The cut-off values of the various test autoantibodies recommended in the product inserts were evaluated according to ROC (receiver operating characteristic) analysis, based on results obtained from RA patients, relative to those of healthy control subjects and patients with SLE or scleroderma.

Plot analysis of ROC curves showed that anti- MCV had a AUC (area under curve) of 0.956 (95\% CI, 0.924-0.988), 0.801 (95\% CI, 0.730-0.872) for anti-CEP-1, 0.912 (95\% CI, 0.861-0.963) for anti-Sa and anti-CCP-IgG 0.929 (95\% CI, 0.8900.968). Furthermore, the EOC (empirical optimal cut-off) was calculated as 91 for anti- MCV with a sensitivity 0.86 and specificity of 0.98 at cut-off. For anti-CEP-1 EOC was 46 with a sensitivity 0.72 and specificity of 0.79 at cut-off. Anti-Sa and anti-CCP-IgG EOC matched the commercially suggested cut-offs of 10 , with a sensitivity 0.86 and 0.87 as well as a specificity of 0.89 and 0.86 at cut-offs for Anti-Sa and anti-CCP-IgG, respectively.

Anti-CCP-IgG and anti-Sa cut-offs concurred with those of the suppliers, while the recommended commercial cut-off values for anti-CEP-1 and anti-MCV were adjusted to $46 \mathrm{RU} / \mathrm{ml}$ and $91 \mathrm{U} / \mathrm{ml}$ respectively and applied in determining further indicators as discussed below and shown in table 3.

Table 3. Sensitivities, specificities, positive predictive values (PPVs), negative predictive values (NPVs) and likelihood ratios (LR) of the various autoantibodies

\begin{tabular}{|c|c|c|c|c|c|c|c|}
\hline & $\begin{array}{l}\text { Sensitivity } \\
\%(95 \% \text { CI })\end{array}$ & $\begin{array}{c}\text { Specificity } \\
\%(95 \% \text { CI })\end{array}$ & $\begin{array}{c}\text { PPV } \\
\%(95 \% \mathrm{CI})\end{array}$ & $\begin{array}{c}\text { NPV } \\
\%(95 \% \text { CI })\end{array}$ & Odds ratio & $+\mathrm{LR}$ & $-\mathbf{L R}$ \\
\hline $\mathrm{cRF}$ & 87 (77-93) & $91(83-96)$ & $89(80-95)$ & $87(80-94)$ & 63 & 9.3 & 0.2 \\
\hline Anti-CCP-IgG & 87 (77-93) & $86(77-92)$ & $83(73-91)$ & $90(81-95)$ & 40 & 6.3 & 0.2 \\
\hline Anti-Sa & $82(72-90)$ & 89 (75-97) & $94(85-98)$ & $72(57-84)$ & 39 & 7.6 & 0.2 \\
\hline Anti-CEP-1 & $72(60-82)$ & $76(65-85)$ & $75(63-85)$ & $73(62-83)$ & 9 & 3.0 & 0.4 \\
\hline Anti-MCV & $86(75-93)$ & $98(92-100)$ & $97(89-100)$ & $90(82-95)$ & 260 & 38.5 & 0.2 \\
\hline
\end{tabular}

$c R F$ composite rheumatoid factor, $C C P$ cyclic citrullinated peptide (IgG), $C E P$ - 1 citrullinated $\alpha$-enolase peptide- $1, M C V$ mutated cyclic vimentin

Highest results achieved in each of the columns are in bold

The sensitivities, specificities, positive predictive values (PPVs), negative predictive values (NPVs), odds ratios and positive (+), negative (-) likelihood ratios (+LR, -LR) for cRF, anti-CCP-IgG, anti-Sa, anti-CEP-1 and anti-MCV are shown in Table 3 and demonstrate that cRF and anti-CCP-IgG are the most sensitive with respect to prediction of RA. Anti-MCV had the best overall specificity and PPV (>95\%) of all autoantibodies tested, as well as the highest + LR value of 260 compared to 9.3 and 6.3 for cRF and anti-CCP-IgG, respectively. 
A matrix correlation between the citrullinated autoantibody titres indicated that anti-CCP-IgG values showed good correlations with anti-MCV and anti-CEP-1 (rho $=0.712, \mathrm{p}<0.000$ and rho $=0.390, \mathrm{p}=0.003$, respectively). AntiMCV and Anti-CEP-1 were also significantly inter-correlated (rho $=0.374, p=0.006$ ).

\section{Disease severity}

a) DAS28-3 and SDAI scores

DAS28-3(CRP) and SDAI scores at first presentation were used as indicators of disease severity. Baseline DAS28-3 scores revealed that $6(8 \%), 27(36 \%)$ and $42(56 \%)$ of RA patients had inactive, moderately active and very active disease, respectively. SDAI at baseline indicated that $25 \%(\mathrm{n}=19)$ and $75 \%(\mathrm{n}=56)$ of patients respectively had HDA, while no patients had LDA or were in remission. DAS28-3 and SDAI scores showed a very significant correlation (rho $=0.866$, $\mathrm{p}<0.000)$. In addition, the classification categories of the 2 different clinical scoring systems were highly compatible according to Fishers' Exact test ( $\mathrm{p}>0.000)$.

None of the 3 citrullinated autoantibody titres under investigation showed statistical significant correlations with the classic disease markers, DAS28-3, SDAI and disease duration individually. However, when combined RF and anti-CCP-IgG positivity was introduced as an additional contributory factor, anti-Sa titers were significantly elevated for DAS28-3 $(\mathrm{p}=0.039), \operatorname{SDAI}(\mathrm{p}=0.019)$ and disease duration $(\mathrm{p}=0.024)$, (Table 4). Furthermore, baseline anti-Sa titres were the highest in the setting of disease duration of 6 months or less when compared with the corresponding median values at 6 to 12 months disease duration $(\mathrm{p}=0.010)$ or longer $(\mathrm{p}=0.024)$ as shown in Figure 1.

Table 4. Associations of anti-Sa autoantibody titers with disease scores when RF and anti-CCP-IgG are considered positive

\begin{tabular}{|lll|}
\hline \hline \multicolumn{1}{|c}{ Disease markers } & Anti-Sa titers $(\boldsymbol{n}=\mathbf{7 5})$ & If RF and anti-CCP-IgG are positive $(\boldsymbol{n}=\mathbf{6 3})$ \\
\hline DAS28-3 & $p=0.069$ & $p=0.039$ \\
SDAI & $p=0.108$ & $p=0.019$ \\
Duration & $p=0.172$ & $p=0.024$ \\
\hline
\end{tabular}




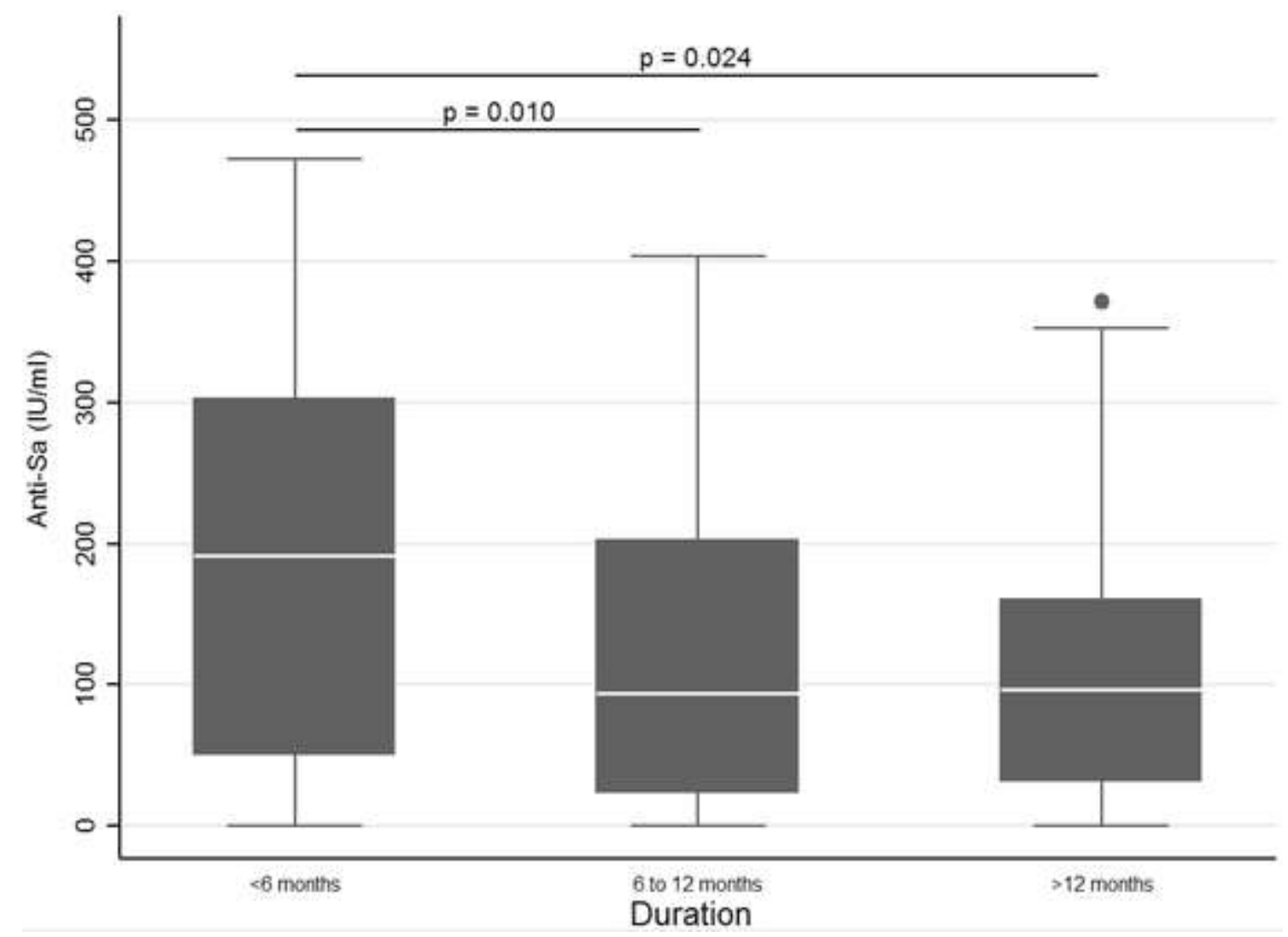

Fig. 1 . Effect of therapy on serum levels of anti-Sa autoantibodies, demonstrating higher titers on presentation which decline significantly on therapy

Anti-CEP-1 positivity significantly, albeit weakly, with the SDAI classification ( $\mathrm{p}=0.046)$; in this context, $81 \%(\mathrm{n}=43)$ of the patients classified with HDA had elevated levels of anti-CEP-1.

\section{Risk factors}

a) HLA-SE

Seventy-one (95\%) RA patients had an HLA-SE allele, of which 30 (40\%) were heterozygous (SX) i.e. had a single HLASE-associated allele, and 55\% where homozygous (SS) for HLA-SE. Only 4 (5\%) of the patients expressed no HLA-SE phenotype (XX) (Table 1). Further classification according to the SE sequence and modulation thereof by amino acid sequences of the HLA-DRB1 gene showed that the 71 patients with HLA-SE alleles could be subdivided into HR [n=48, $(68 \%)]$ and LR groups $[\mathrm{n}=23,(31 \%)]$.

As shown in Figure 2, statistically significant differences in the serum levels of anti-CCP-IgG ( $p=0.009)$, anti-Sa $(\mathrm{p}=0.026)$ and anti- CEP-1 ( $\mathrm{p}=0.001)$ antibodies were noted when comparing the SS vs. SX HLA-SE allele expression groups, with the homozygous (SS) patients having consistently higher median autoantibody titres than their heterozygous (SX) counterparts. This was not apparent in the case of anti-MCV. 


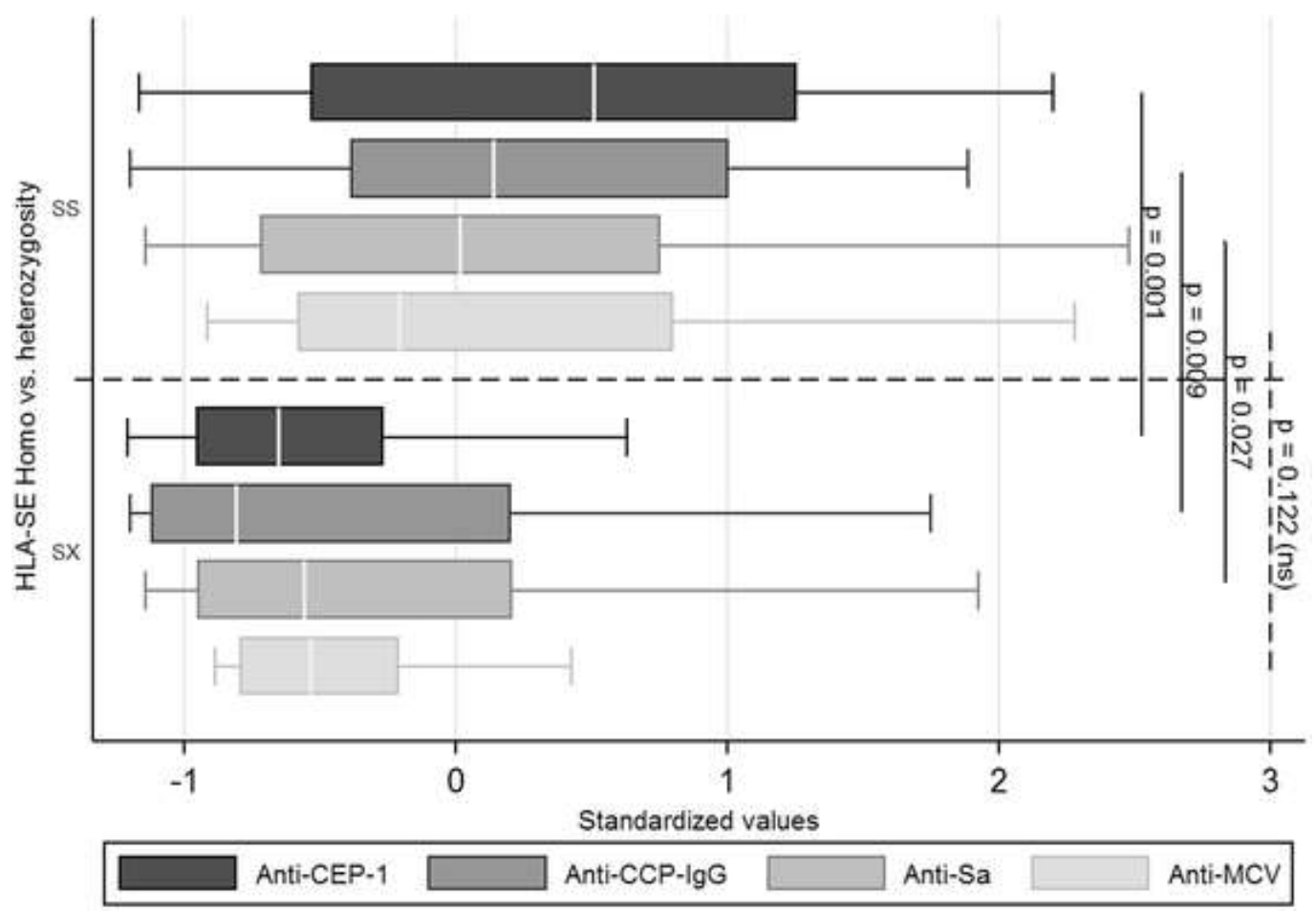

Fig. 2. Relationships between anti-CEP-1, anti-CCP-IgG, anti-Sa and anti-MCV titers, and HLA-SE expression [homozygous (SS) vs. heterozygous $(\mathrm{SX})] . n s$ not statistically significant

Comparing the HR vs. LR groups revealed that only median anti-Sa titres were significantly higher $(p=0.012)$ in the HR group compared to the LR group. Anti-CEP-1 bordered on statistical significance $(\mathrm{p}=0.052)$ and anti-CCP-IgG $(\mathrm{p}=0.074)$ showed the same trend as shown in Figure 3.

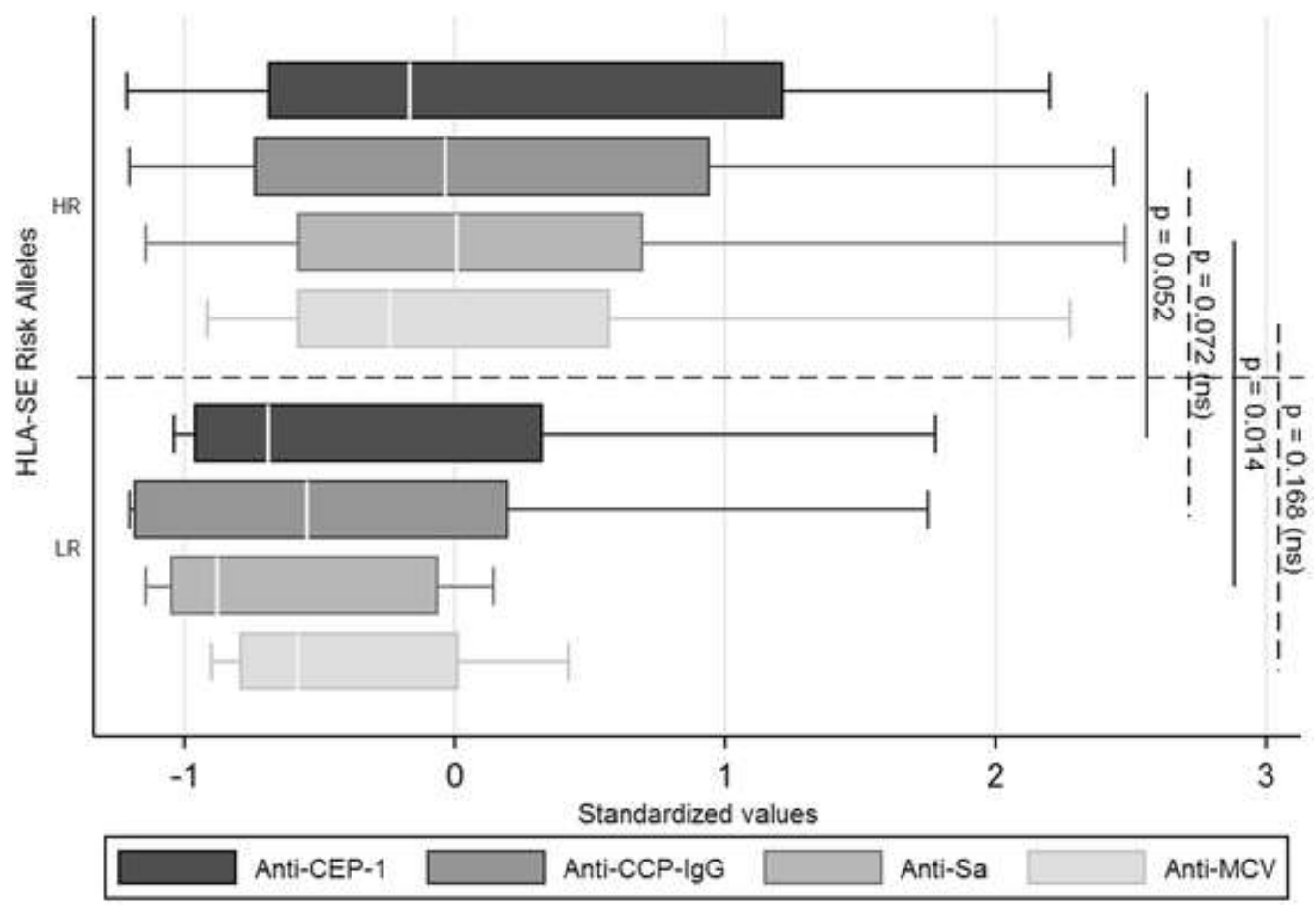

Fig. 3. Relationships between anti-CEP-1, anti-CCP-IgG, anti-Sa and anti-MCV titers, and HLA-SE risk-associated alleles [high risk (HR) vs. Low Risk (LR)]. ns not statistically significant 


\section{b) Tobacco use}

Tobacco use, according to serum cotinine levels in excess of $3.0 \mathrm{ng} / \mathrm{ml} .[13]$, was detected in 34 (45\%) patients and as shown in Figure 4 these patients presented with statistically higher SDAI ( $\mathrm{p}=0.031)$ and DAS28-3 ( $\mathrm{p}=0.039)$ scores at baseline (using the Mann-Whitney test).
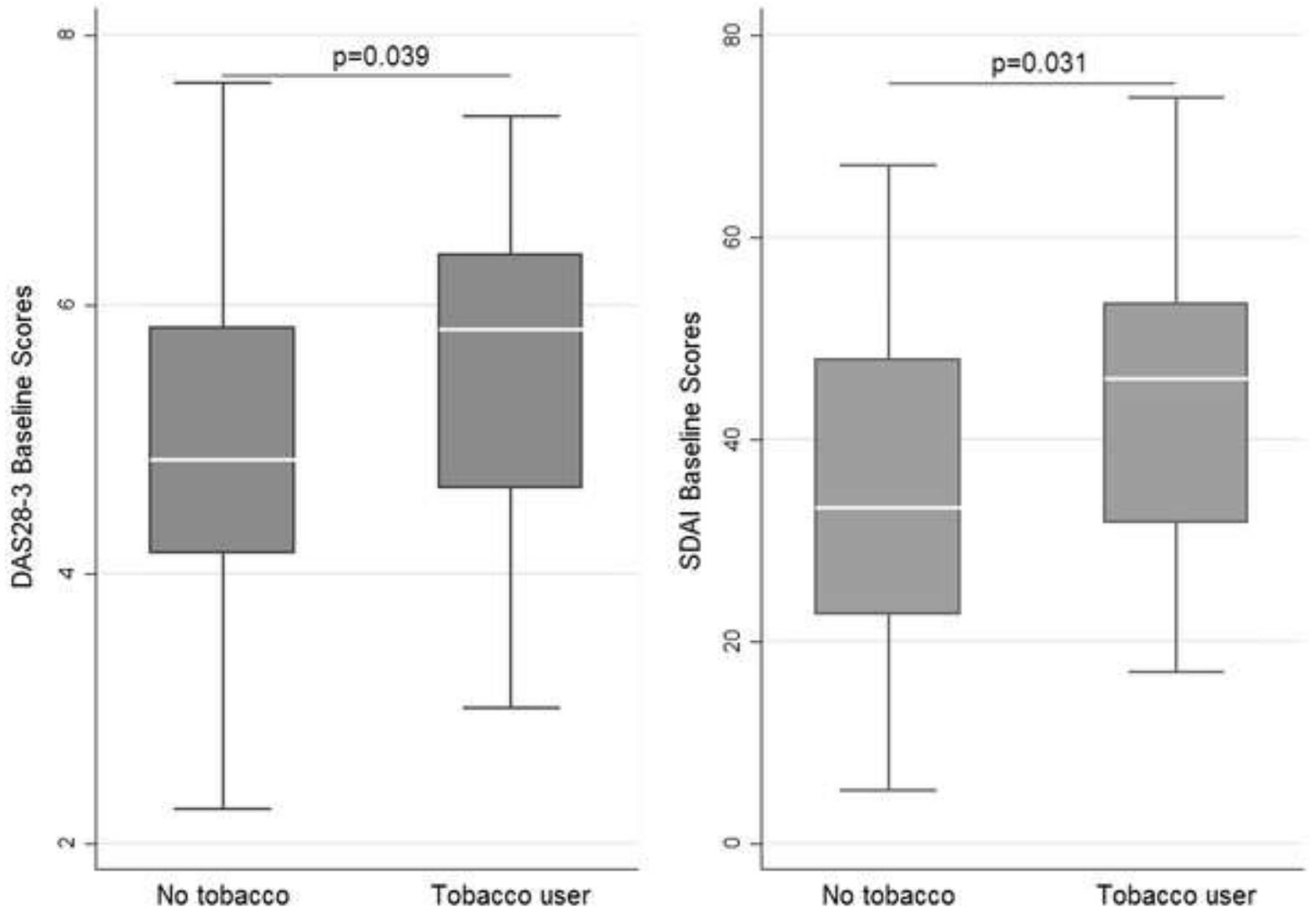

Fig. 4. Box and Whisker plots reflecting SDAI and DAS $28-3$ baseline scores compared with tobacco use $(<3.0 \mathrm{ng} / \mathrm{ml}=\mathrm{no}$ tobacco use)

\section{Response to treatment}

a) SDAI response

The response to treatment was determined as a percentage increase / decrease in SDAI when baseline scores were compared to SDAI scores at 6 months. Categories were defined from the original suggested SDAI response categories (SDAI 50\%, 70\%, 85\% or minor, moderate and major responses) [14]. Of 67 patients for whom both baseline and 6 months SDAI scores were recorded, $30(44.8 \%)$ patients did not achieve a minor change $(<50 \%), 17(28.4 \%)$ attained a minor (50-70\%), $10(14.9 \%)$ a moderate $(70-85 \%)$ and $10(14.9 \%)$ a major response (>85\%). SDAI responses were further classified as binary, with either no response $(<50 \%)$ or any response $(>50 \%)$, according to which $30(44,8 \%)$ patients showed no response vs. 37(55.2\%) who had a response.

Comparison of the titres of the different citrullinated autoantibodies in relation to the SDAI categorical response using the Kruskal-Wallis test revealed a statistically significant association of anti-MCV titres with SDAI ( $\mathrm{p}=0.031$ ), while the SDAI binary response classification indicated that elevated titres of anti-CCP- $\operatorname{IgG}(\mathrm{p}=0.027)$ and anti-MCV $(\mathrm{p}=0.003)$ antibodies 
at presentation were associated with the SDAI response. These associations, particularly in the case of anti-CCP-IgG, were strongest in the case of tobacco users, the levels of statistical significance being $\mathrm{p}=0.007$ and $\mathrm{p}=0.002$ for anti-CCP-IgG and anti-MCV antibodies respectively. Similar associations were not, however, detected in the case of non-tobacco users ( $\mathrm{p}=0.413$ and $\mathrm{p}=0.129$ for anti-CCP-IgG and anti-MCV respectively).
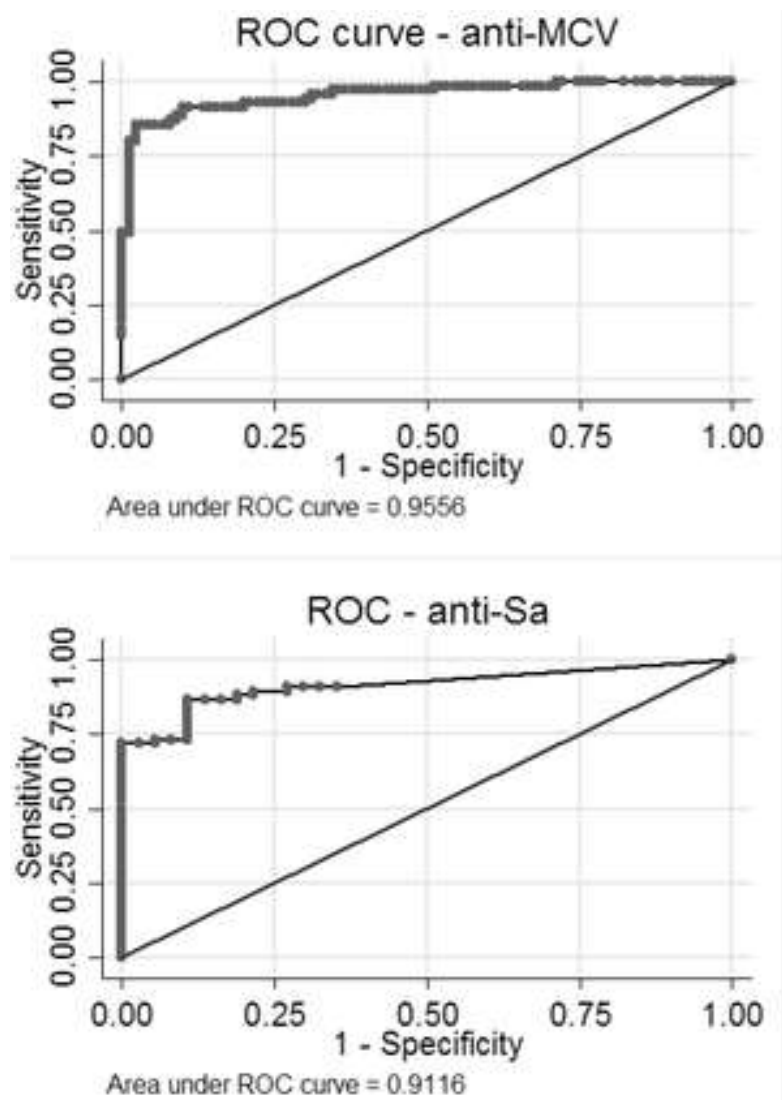

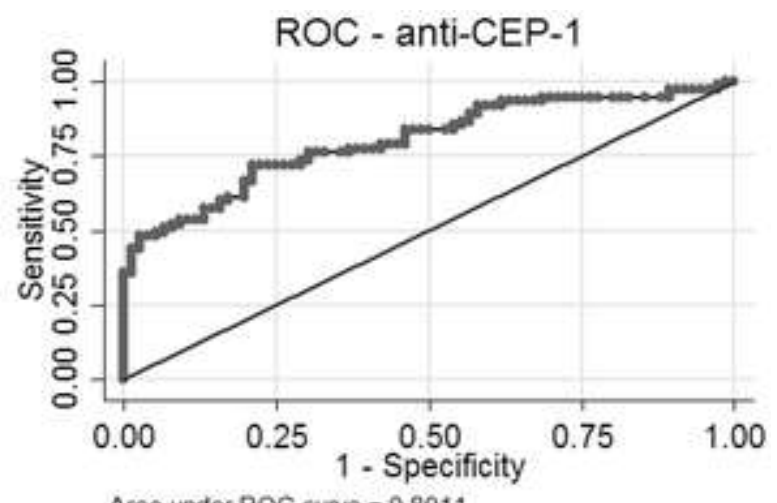

Area under $R O C$ curve $=0.8011$

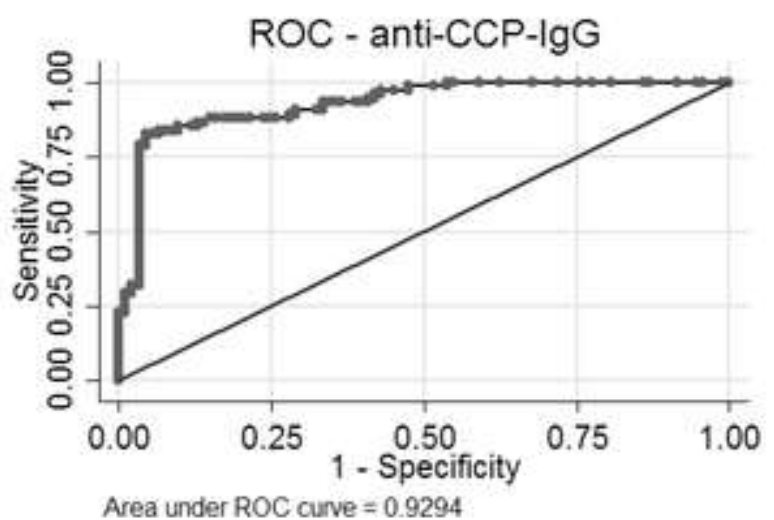

Fig. 5. ROC curves for anti-MCV, anti-CEP-1, anti-Sa and anti-CCP-IgG

\section{Discussion}

In the current study, elevated levels of anti-Sa showed an association with higher disease activity as previously reported [15, 16], as well as with HLA-SE homozygosity and high-risk alleles. Interestingly, this finding of an association of anti-Sa autoantibodies with genetic predisposition and high risk have not, to our knowledge, been previously described, possibly justifying the inclusion of anti-Sa in the profile of serodiagnostic tests for our RA population. In this context, it is noteworthy that anti-Sa is directed against citrullinated vimentin [17] which has been shown in both murine experimental models and in humans with RA to bind to HLADRB1*0401[18].

Associations of anti-CEP-1 antibodies with disease severity and HLA-SE, as well as with smoking, have been reported previously, mostly in RA patients of Caucasian origin [19-21], and, according to the findings of the current study, these associations are also evident in RA patients of African origin. The association with HLASE motif is underscored not only in the case of RA patients who present as homozygous for the HLA-SE 
genotype, but also those with high-risk alleles, an observation, which, to our knowledge, has not been described previously.

In the case of anti-MCV, measurement of this autoantibody demonstrated impressive specificity, positive predictive value and a positive likelihood ratio, as well as significant associations with SDAI response especially with tobacco use, indicative of its potential to act as the preferred RA serodiagnostic procedure in our setting. Although the diagnostic potential of anti-MCV, as well as its role in the immunopathogenesis of RA, are well-described [22, 23], as are its associations with other ACPAs, HLA-SE and smoking [24-27], measurement of this autoantibody in the cohort of RA patients described in the current study appears to be of particular diagnostic value, exceeding the predictive odds ratio of any other ACPA test by four-fold. However, this finding has to be tempered by a consideration of the adjusted cut-off value, warranting further testing with larger cohorts.

In conclusion, measurement of anti-Sa as an additional biomarker in the routine serodiagnosis/prognosis of RA may have added value, albeit limited, in the context of assessment of disease severity. Most importantly, however, anti-MCV is shown to be equivalent to, possibly even exceeding, the serodiagnostic potential of any of the other autoantibodies tested in the cohort of South African patients investigated in this study.

\section{References}

1. Aletaha D, Neogi T, Silman AJ, Funovits J, Felson DT, Bingham CO, 3rd, et al. 2010 Rheumatoid arthritis classification criteria: an American College of Rheumatology/European League Against Rheumatism collaborative initiative. Arthritis Rheum. 2010;62(9):2569-2581.

2. Aggarwal R, Liao K, Nair R, Ringold S, Costenbader KH. Anti-citrullinated peptide antibody assays and their role in the diagnosis of rheumatoid arthritis. Arthritis Rheum. 2009;61(11):1472-1483.

3. Deane KD. Preclinical rheumatoid arthritis (autoantibodies): an updated review. Curr Rheumatol Rep. 2014;16(5):419.

4. Hu ZD, Deng AM. Autoantibodies in pre-clinical autoimmune disease. Clin Chim Acta. 2014;437:14-18.

5. Meyer PW, Ally MM, Anderson R. Reliable and cost-effective serodiagnosis of rheumatoid arthritis. Rheumatol Int. 2016;36(6):751-758.

6. Meyer PW, Hodkinson B, Ally M, Musenge E, Wadee AA, Fickl H, et al. Circulating cytokine profiles and their relationships with autoantibodies, acute phase reactants, and disease activity in patients with rheumatoid arthritis. Mediators Inflamm. 2010;2010:158514.

7. Meyer PW, Hodkinson B, Ally M, Musenge E, Wadee AA, Fickl H, et al. HLA-DRB1 shared epitope genotyping using the revised classification and its association with circulating autoantibodies, acute phase reactants, cytokines and clinical indices of disease activity in a cohort of South African rheumatoid arthritis patients. Arthritis Res Ther. 2011;13(5):R160.

8. Ally MM, Hodkinson B, Meyer PW, Musenge E, Tintinger GR, Tikly M, et al. Circulating anti- 
citrullinated peptide antibodies, cytokines and genotype as biomarkers of response to disease-modifying antirheumatic drug therapy in early rheumatoid arthritis. BMC Musculoskelet Disord. 2015;16:130.

9. Aletaha D, Smolen J. The Simplified Disease Activity Index (SDAI) and the Clinical Disease Activity Index (CDAI): A review of their usefulness and validity in rheumatoid arthritis. Clinical and Experimental Rheumatology. 2005;23(5):S100-S108.

10. Fransen J, Stucki G, Van Riel PLCM. Rheumatoid arthritis measures: Disease Activity Score (DAS), Disease Activity Score-28 (DAS28), Rapid Assessment of Disease Activity in Rheumatology (RADAR), and Rheumatoid Arthritis Disease Activity Index (RADAI). ARTHRITIS \& RHEUMATISM. 2003;49(S5):S214S224.

11. Tezenas Du Montcel S, Michou L, Petit-Teixeira E, Osorio J, Lemaire I, Lasbleiz S, et al. New classification of HLA-DRB1 alleles supports the shared epitope hypothesis of rheumatoid arthritis susceptibility. Arthritis and Rheumatism. 2005;52(4):1063-1068.

12. Michou L, Croiseau P, Petit-Teixeira E, Du Montcel ST, Lemaire I, Pierlot C, et al. Validation of the reshaped shared epitope HLA-DRB1 classification in rheumatoid arthritis. Arthritis Res Ther. 2006;8(3):R79.

13. Benowitz NL, Bernert JT, Caraballo RS, Holiday DB, Wang J. Optimal serum cotinine levels for distinguishing cigarette smokers and nonsmokers within different racial/ethnic groups in the United States between 1999 and 2004. Am J Epidemiol. 2009;169(2):236-248.

14. Aletaha D, Martinez-Avila J, Kvien TK, Smolen JS. Definition of treatment response in rheumatoid arthritis based on the simplified and the clinical disease activity index. Ann Rheum Dis. 2012;71(7):11901196.

15. Hou YF, Sun GZ, Sun HS, Pan WP, Liu WB, Zhang CQ. Diagnostic value of anti-Sa and anticitrullinated protein antibodies in rheumatoid arthritis. Journal of Rheumatology. 2012;39(8):1506-1508.

16. Iwaszkiewicz C, Puszczewicz M, Bialkowska-Puszczewicz G. Diagnostic value of the anti-Sa antibody compared with the anti-cyclic citrullinated peptide antibody in rheumatoid arthritis. Int $J$ Rheum Dis. 2015;18(1):46-51.

17. Vossenaar ER, Despres N, Lapointe E, Van Der Heijden A, Lora M, Senshu T, et al. Rheumatoid arthritis specific anti-Sa antibodies target citrullinated vimentin. Arthritis Research \& Therapy. 2004;6(2):R142-150.

18. Snir O, Rieck M, Gebe JA, Yue BB, Rawlings CA, Nepom G, et al. Identification and functional characterization of $\mathrm{T}$ cells reactive to citrullinated vimentin in HLA-DRB1*0401-positive humanized mice and rheumatoid arthritis patients. Arthritis Rheum. 2011;63(10):2873-2883.

19. Mahdi H, Fisher BA, Kallberg H, Plant D, Malmstrom V, Ronnelid J, et al. Specific interaction between genotype, smoking and autoimmunity to citrullinated [alpha]-enolase in the etiology of rheumatoid arthritis. Nat Genet. 2009;41(12):1319-1324.

20. Wegner N, Lundberg K, Kinloch A, Fisher B, Malmström V, Feldmann M, et al. Autoimmunity to specific citrullinated proteins gives the first clues to the etiology of rheumatoid arthritis. Immunological reviews. 2010;233(1):34-54.

21. Fisher BA, Plant D, Brode M, Van Vollenhoven RF, Mathsson L, Symmons D, et al. Antibodies to 
citrullinated alpha-enolase peptide 1 and clinical and radiological outcomes in rheumatoid arthritis. Ann Rheum Dis. 2011;70(6):1095-1098.

22. Dejaco C, Klotz W, Larcher H, Duftner C, Schirmer M, Herold M. Diagnostic value of antibodies against a modified citrullinated vimentin in rheumatoid arthritis. Arthritis Research \& Therapy. 2006;8(4):R119.

23. Bang H, Egerer K, Gauliard A, Luthke K, Rudolph PE, Fredenhagen G, et al. Mutation and citrullination modifies vimentin to a novel autoantigen for rheumatoid arthritis. Arthritis Rheum. 2007;56(8):2503-2511.

24. Luime JJ, Colin EM, Hazes JMW, Lubberts E. Does anti-mutated citrullinated vimentin have additional value as a serological marker in the diagnostic and prognostic investigation of patients with rheumatoid arthritis? A systematic review. Annals of the Rheumatic Diseases. 2010;69(2):337-344.

25. Diaz-Toscano ML, Olivas-Flores EM, Zavaleta-Muniz SA, Gamez-Nava JI, Cardona-Munoz EG, PonceGuarneros M, et al. Comparison of two assays to determine anti-citrullinated peptide antibodies in rheumatoid arthritis in relation to other chronic inflammatory rheumatic diseases: assaying anti-modified citrullinated vimentin antibodies adds value to second-generation anti-citrullinated cyclic peptides testing. Biomed Res Int. 2014;2014:198198.

26. Reyes-Castillo Z, Palafox-Sanchez CA, Parra-Rojas I, Martinez-Bonilla GE, Del Toro-Arreola S, Ramirez-Duenas MG, et al. Comparative analysis of autoantibodies targeting peptidylarginine deiminase type 4, mutated citrullinated vimentin and cyclic citrullinated peptides in rheumatoid arthritis: associations with cytokine profiles, clinical and genetic features. Clin Exp Immunol. 2015;182(2):119-131.

27. Sun P, Wang W, Chen L, Li N, Meng X, Bian J, et al. Diagnostic value of autoantibodies combined detection for rheumatoid arthritis. J Clin Lab Anal. 2017;31(5). 